\title{
Akustisches Gedächtnis und bruitistisches Gedenken an den Ersten Weltkrieg: das Album Lament der Einstürzenden Neubauten (2014)
}

\author{
Andreas Häcker
}

\section{Abstract}

Zum Gedenken an den Ersten Weltkrieg wurden in Belgien einzigartige Musikwerke in Auftrag gegeben wie Lament, Ypres oder Shell Shock, a requiem of war, eine Oper über das posttraumatische Syndrom der Frontsoldaten. Für die Gedenkfeiern in Westflandern hat die Berliner Experimental-Band Einstürzende Neubauten um Blixa Bargeld die Performance Lament im Auftrag der Regierung Westflanderns konzipiert. Lament erschien zunächst als Studioalbum, die Weltpremiere fand am 8. November 2014 in Diksmuide statt, es folgte alsbald eine Tournee mit rund 30 Konzerten in Europa und Australien bis 2018. Obwohl die Wahl deutscher Underground-Musiker verwirrend für manchen belgischen Musikkritiker sein mochte, ist das Album durch eine bedeutende Recherchearbeit geprägt: Es verbindet bruitistische Komposition und historisches Material aus Tonarchiven. Ziel dieser Arbeit ist es zu untersuchen, welchen Beitrag die Musiker zur Erinnerungsarbeit an den Ersten Weltkrieg leisten, welche Herausforderungen ein Auftragswerk stellt und wie die Erwartungen des Publikums erfüllt werden.

Les commémorations de la Première Guerre mondiale en Belgique ont encouragé la création d'œuvres musicales singulières comme Lament, Ypres ou Shell Shock, a requiem of war, opéra autour du syndrome post-traumatique dont souffrent les combattants. Le groupe expérimental berlinois Einstürzende Neubauten et leur chanteur Blixa Bargeld conçoivent la performance Lament d'après une commande gouvernementale pour les commémorations en Flandre occidentale. Cet album est d'abord produit en studio et présenté en concert, en première mondiale, à Dixmude le 8 novembre 2014, puis le groupe part en tournée, avec une trentaine de dates, en Europe et en Australie jusqu'en 2018. Bien que ce choix d'un groupe allemand puisse paraître surprenant pour certains critiques belges, l'album comporte un travail de recherche important et combine un savoir-faire bruitiste et un travail sensible avec les archives sonores historiques issues de la Première Guerre mondiale. Cet article vise à étudier les apports des musiciens, les enjeux d'une œuvre de commande et les attentes des publics.

The commemorations of the First World War in Belgium encouraged the creation of singular musical works such as Lament, Ypres or Shell Shock, a requiem of war, opera around the post-traumatic syndrome suffered by the combatants. The Berlin experimental group Einstürzende Neubauten and its frontman Blixa Bargeld designed Lament after a commission from the West Flemish government for the commemoratives in West Flanders. This album was produced in the studio, then world premiered in Dixmude on November 8, 2014. The group then toured Europe and Australia until 2018, with around 30 dates. Although this choice of a German band may seem embarrassing to some Belgian critics, the album includes a significant amount of research work and combines noise expertise with historical sound archives from the First World War. This article aims to study the contributions of the musicians, the stakes of a commissioned work and the expectations of the public.

\section{Keywords}

Erster Weltkrieg • Einstürzende Neubauten • Erinnerungsarbeit • Auftragswerk • Tonarchive

Première Guerre mondiale • Einstürzende Neubauten • commémoration • œuvre de commande • archives sonores

First World War • Einstürzende Neubauten $\bullet$ commemoration $\bullet$ commissioned work $•$ sound archives

Vor eminenten Jahrestagen beauftragt man gerne bildende und darstellende Künstler·innen: Sie sollen zu Gedenkfeierlichkeiten (be)deutende Werke schaffen. Der Erste Weltkrieg schlug Wunden, die Erinnerung und das Gedenken an inn können viele Fragen an die Kunstschaffenden stellen: Wie soll man mit den Traumata umgehen und wie viel Stille ist angebracht? Mit welchen historischen Quellen ist zu arbeiten? Muss man auf die Permanenz von Kriegen hinweisen? Jens
Utthoff (2014) erkennt ein zentrales Problem und fragt: „Wie kann man Erinnerung erneuern?"

In Vorbereitung auf die Jahre 2014-2018, in denen an den Beginn, Verlauf und das Ende des Ersten Weltkriegs vor hundert Jahren gedacht wurde, orderte man in Belgien mehrere beachtenswerte Musikwerke. Das Museum In Flanders Field baute um und gab 2011 der britischen Indierock-Band Tindersticks den Auftrag, eine Klanglandschaft für die neue 
Dauerausstellung zur Wiedereröffnung im Juni 2012 zu schaffen. Der museale Soundtrack erschien 2014 als Album unter dem Titel Ypres. ${ }^{1}$ Das Opernhaus La Monnaie engagierte seinerseits den in Ypern geborenen belgischen Komponisten Nicholas Lens und als Librettisten den australischen Poeten und Popmusiker Nick Cave. Gemeinsam arbeiteten beide an der Oper Shell shock, a requiem of war, deren Uraufführung am 24. Oktober 2014 in Brüssel stattfand. Vier Jahre später wurde sie in Paris aufgeführt. ${ }^{2}$

Das Album Lament der Band Einstürzende Neubauten soll hier nun auf die Probleme eines Auftragswerks allgemein und eines Kunstwerkes zum Kriegsgedenken untersucht werden. Bei unserer Studie der Werkgenese konzentriert sich der Blick auf die kreativen Prozesse, institutionnellen Zusammenhänge und Erwartungen des Publikums. Lament wurde zwischen 2013 und 2014 als Studioalbum geschaffen, am 8 . November 2014 war seine Weltpremiere im Busdepot von Diksmuide, zum Gedenken an die Zerstörung der Stadt in Westflandern. Nach einer Tournee durch Europa und nach Australien mit rund 30 Aufführungsterminen traten die Einstürzenden Neubauten mit Lament einmal beim Abschlusswochenende eines mehrjährigen Kulturprogramms in Ypern im August 2018 und dreimal im Berliner Pierre-Boulez-Saal im November 2018 auf.

\section{DER AUFTRAG AUS DIKSMUIDE}

Beginnen wir mit dem Kontext des Auftrags aus Diksmuide und den reichen Erfahrungen der Berliner Band mit historischen Stätten. Album, Uraufführung und weitere Konzerte ${ }^{3}$ geben einen guten Einblick in Wege einer sensiblen und aufmerksamen Erinnerungsarbeit. In Westflandern ist die Landschaft vernarbt, überall sind Soldatenfriedhöfe zu finden. Auf einem von innen, in der Nähe von Diksmuide, liegt Peter Kollwitz (18861914), Sohn der Bildhauerin Käthe Kollwitz. ${ }^{4}$ Die Schlachten waren grauenvoll, endeten oft im Nahkampf, Mann gegen Mann. Bei der Schlacht an der Yser wurde Ende Oktober 1914 das Land geflutet, um die deutsche Armee aufzuhalten. Nach langen Zusammenstößen mit französischen Soldaten nahmen deutsche Truppen Diksmuide am 10. November 1914 ein. Die

Tindersticks (2014), Ypres, Berlin, Lucky Dog/City Slang, 2014. Die Musiker berücksichtigten für ihre Komposition die Architektur des Museums. Da die Räume „innerhalb der Tuchhalle" in der Tonart F zu schwingen schienen, so das Museum auf seiner Internetseite, sei die Musik in einem Cluster mit Es, F und Fis ausgearbeitet worden. Die orchestrale Klanglandschaft wurde so zusammengemischt, dass sie den ganzen Tag in Endlosschleife in den Ausstellungsräumen gehört werden kann.

2 Shell Shock erschien 2016 als CD bei Universal Music Belgium. Im Rahmen des 100 Jahrestags der Armistice wurde die Oper am 11. November 2018 in der Cité de la musiquePhilharmonie de Paris wiederaufgeführt. Der Fernsehsender arte hat beide Aufführunge übertragen; noch heute kann man den Pariser Mitschnitt sehen, als DVD (Paris, LGM, 2018) oder im Youtube-Kanal von France Musique (https://youtu.be/F3bGhqROG8E, zuletzt aufgerufen am 3. April 2021).

3 Einstürzende Neubauten (2014), Lament, London, Mute. Dieser Artikel konzentriert sich auf das Album. Konzertkritiken sowie Amateurvideos gaben Einblicke in die LivePerformance. Besonderer Dank geht an Anna Szczuka von den Einstürenden Neubauten, Performance. Besonderer Dank geht an Anna Szczuka von den Einstürzenden Neubauten, die für die Untersuchung der Rezeption einen umfassenden Pressespiegel zur Verfügun gestellt hat, der durch eine Recherche in Online-Archiven belgischer und deutscher Medien, darunter Fanzines, ergänzt wurde. Wichtige Quellen zur Werkgenetik sind zahlreiche Interviews Blixa Bargelds, das Booklet der CD-Fassung, das Programmheft zur Uraufführung sowie das Saalheft, das der Pierre-Boulez-Saal in Berlin für die Konzerte im November 2018 herausgegeben hat.

4 Der Tod des Sohnes erscheint oft in Werken von Käthe Kollwitz. Siehe dazu den Beitrag von Joachim Rees in dieser Nummer.
Stadt wurde während des Krieges verwüstet. Den Ausgang der ersten Flandernschlacht und die Zahl der Toten während der ersten Kriegsmonate umreißt der französische Historiker und ehemalige Offizier Michaël Bourlet so:

La fin des batailles de l'Yser et d'Ypres met un terme à la guerre de mouvement et le début de la guerre de position. La stabilisation du front occidental s'achève par des combats acharnés dans la Flandre belge. Les Allemands échouent à atteindre la Manche. Quant aux Franco-Britanniques, ils ne réalisent pas la percée tant attendue mais réussissent néanmoins à contenir l'avance allemande au prix des pertes considérables. [...] Les principales villes, Nieuport et Dixmude, sont dévastées, comme plus d'une dizaine de villages. Les premiers mois de campagne [...] ont été coûteux pour tous les belligérants. Entre août et novembre 1914, environ 300.000 soldats français, 241.000 soldats allemands, 30.000 Britanniques et 30.000 Belges, soit un total de plus de 600.000 soldats, périssent sur le front de l'ouest. (Bourlet 2012: 81)

Wie arbeitet Kunst nun zum Thema Krieg? Ist nur Wehklage möglich? Gewiss unterliegt die Produktion einer Oper, musealen Beschallung oder eines Performance-Konzertes bestimmten kreativen, aber auch ökonomischen Abläufen; Inhalte und Publikum sind anders als bei einem militärhistorischen Buch. Sinnlichkeit und Sinn stehen vor Frontkarten, Opferlisten und Verluststatistiken. Musik ist eine Mittlerin von Emotionen und Erinnerungen, doch auch statistische und historische Daten können einem Musikwerk subtile Struktur geben, wie wir später sehen werden.

Auch wenn das Thema Krieg kein „Herzanliegen“ (Müller 2014) für den Musiker Blixa Bargeld ${ }^{5}$ war, besuchte er im Sommer 2011 mit seinem Manager, mit dem Bandkollegen Andrew Unruh und ihrem belgischen Booker, Peter Verstraelen, den Muziekclub 4AD und erkundete die Region um Diksmuide. Zwei Jahre später, im August 2013, kam dann ein Auftrag zu einem Konzert, das sich in eine Reihe kultureller Veranstaltungen zum Gedenken an den Ersten Weltkrieg einreihte. Musiker-innen wie Veranstalter-innen hatten freie Hand, wie Marjan Dewulf vom Muziekclub 4AD in Diksmuide berichtet:

This concert was part of a series of commemorative events during 2014-2018, under the title of GoneWest. Music Club 4AD, located in Dixmude, was the artistic and productional partner for this event and proposed to commission Einstürzende Neubauten. [...] They also had a carte blanche from 4AD and GoneWest, there was no artistic interference whatsoever. [...] GoneWest had a clear artistic vision, that was written down in a letter of intent [...] there is a call to rethink the commemoration, to include all nationalities involved and to have a more complex, diverse and contemporary approach to commemoration. This Letter of Intent was approved by the West Flemish

5 Der Name ist Programm: Blixa Bargeld. Schon vor der Gründung der Einstürzenden Neubauten nannte sich so der 1959 in Westberlin geborene Künstler mit Bezug auf eine Filzstiftmarke und eine Hommage an den Maler, Grafiker und Schriftsteller Johannes Theodor Baargeld (1892-1927), der 1919 mit Max Ernst die Dada-Gruppe in Köln gründete (Dax Defcon 2010: 19-20). 
governement and made it possible for the artistic board (including $4 A D$ ) and coordinator of the project to make independent artistic choices. So there was no need to persuade Flemish politicians specifically for Einstürzende Neubauten. ${ }^{6}$

Das GoneWest-Programm sah klar ein Problem: das allmähliche Verschwinden direkter Zeitzeug innen. Doch bot dies auch Möglichkeiten, transnational ${ }^{7}$ Wissen und Empathie zu verbinden, wie man in der Absichtserklärung lesen kann:

The commemoration of the outbreak of World War I will take place one hundred years after the event without any direct witnesses still being alive. The chain of living memories which used to physically bind us to the Great War has gradually been broken. A past that used to be made up of flesh and flood is transitioning towards a memory comprising of stone, ink and paper. This has made it necessary to rethink the commemoration. [...] In order to keep the memory of the Great War alive in all of its complexity, we need to awaken our human capacity for empathy while maintaining a sound historical perspective. A hundred years after the event, we can also enter into a gradual process of remembering without posthumously dividing the dead from the War up into winners and losers, good and bad, civilians and soldiers.

Während im Bereich der Literatur die Macher-innen des Kulturprogramms GoneWest die Arbeiten etablierter Autor $\cdot$ innen und Texte einfacher Leute als Ganzes sahen, so galt es die Musikproduktionen verschiedenster Genres interdisziplinär entlang der historischen Geschehnisse zu organisieren und auch den Pazifismus ins Zentrum zu rücken. In der Absichtserklärung liest man:

[...] the historic timeline will be followed as much as possible by linking music creations to significant events and/or locations where possible. The antiwar philosophy will be of central importance. It will be attempted to come up with creations that belong to different genres.

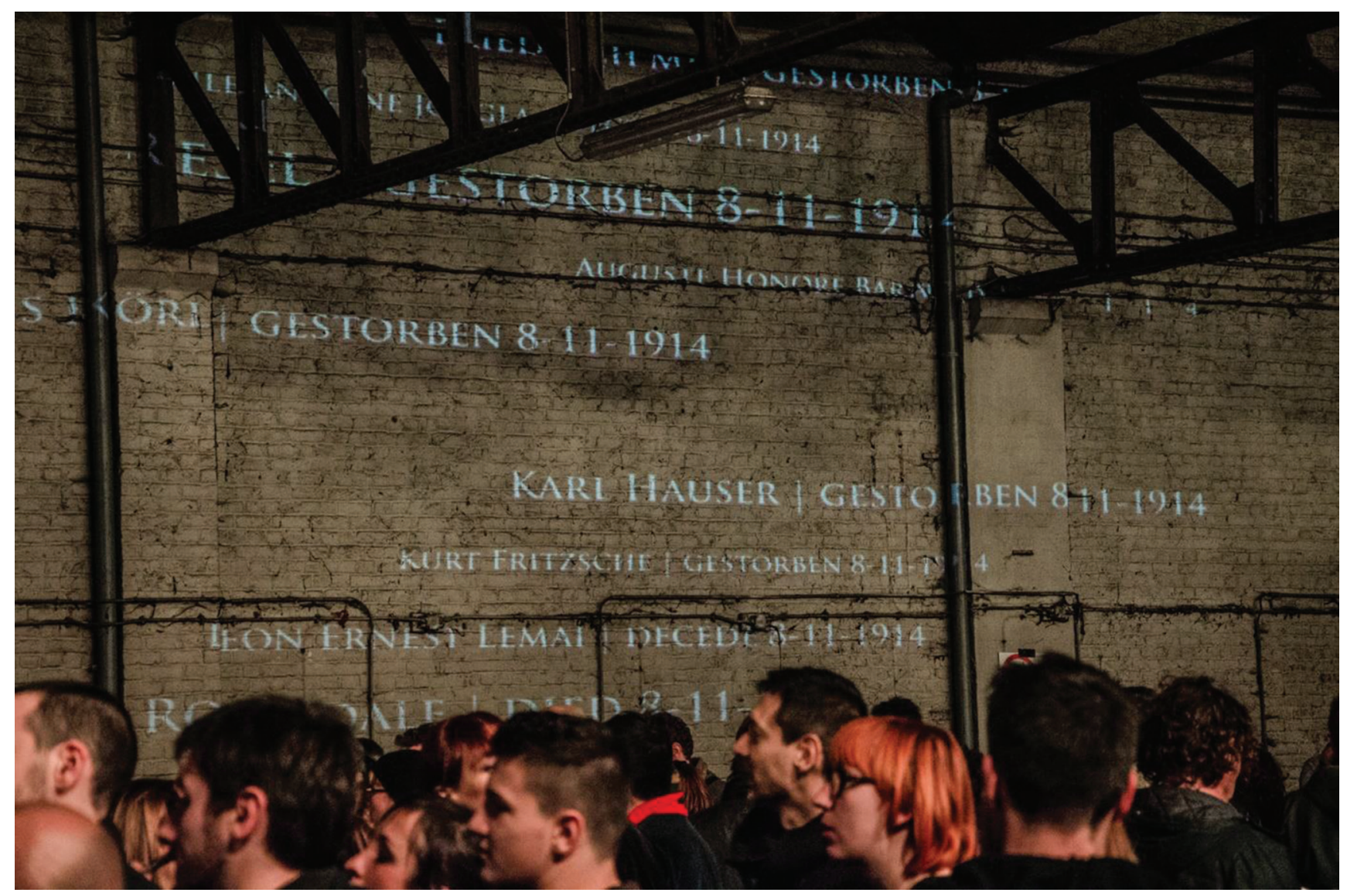

Abb.1: Zu den Veranstaltungen des GoneWest-Erinnerungsprogramms (2014-2018) wurde als Videomapping eine Namensliste der Verstorbenen aller Kriegsnationen in Westhoek ${ }^{8}$ projiziert, hier am Abend der Weltpremiere von Lament, Busdepot Diksmuide, 8. November 2014. () Wannes Dewilde.

6 E-Mail an den Autor dieses Beitrags, 29. November 2019.

7 Xavier Kruth vom Webzine Dark Entries schreibt über den westflämischen Auftrag an eine deutsche Band: ,Germans celebrating the brutal victory and occupation in Diksmuide? Let that be something the organisers definitely do not want to hear." (Kruth 2014)
8 „Für dieses ehemalige Kriegsgebiet“ galt es, „ein umfassendes Register der Opfer zu erstellen, also eine Liste, auf der sowohl Zivilisten als auch Militärangehörige ungeachte ihrer Nationalität verzeichnet sind“; die Namensliste ist heute im Museum In Flanders Fields integriert und als Suchmaschine online zugänglich: https://www.inflandersfields.be/de/kenniscentrum-d/namenlijst-d, zuletzt aufgerufen am 25. Februar 2021 
Als Termin für das Konzert der Einstürzenden Neubauten war der Jahrestag der Verwüstung der Stadt Diksmuide vorgesehen. Im Programmheft ${ }^{9}$ zum Konzert in Diksmuide spricht Blixa Bargeld von den Schwierigkeiten des Themas Krieg, sieht aber rückblickend die Chancen des Auftragswerkes in der Möglichkeit nach überraschenden Aspekten zu suchen:

It was never an idea of my heart to write something about war altogether or the first world war specially. But taking that commission and diving deep into the whole subject, I found some niches and some corners of that subject that were not told before, which was interesting to me.

Sicher sind die Einstürzenden Neubauten Musiker erster Wahl, wenn es um Auftritte an geschichtsträchtigen Orten geht. Nach „einem spontanen Auftritt im Club Moon“ gründete sich die Band 1980 und wurde zu einer Ikone der Westberliner Subkultur (Emmerling/Weh 2014: 38). Die Fans ihres polyglotten und lärmenden CEuvre finde man, so Blixa Bargeld in einem Euronews-Interview, wohl weniger in Deutschland. Nicht ohne Sinn für Humor unterstreicht er enge Verbindungen zwischen Berlin und dem Rest der Welt. Insbesondere Forscher·innen in anglophonen Ländern (z.B. Shryane 2009) beschäftigen sich mit ihrem Werk. ${ }^{10}$ Kreuzberg im amerikanischen Sektor ist inr Zuhause. Der Stadtteil an der Mauer ist markant für die geteilte Stadt und Musik, die eine neue Epoche bewegt: Punk, New-Wave, NDW, bruitistische und experimentelle Musik. Den distinktiven Klang der Band um Blixa Bargeld beschreibt die Kuratorin Mathilde Weh im Katalog der Ausstellung Geniale Dilletanten - Subkultur der 1980er Jahre in Deutschland so:

Anfangs spielten sie ihre Konzerte noch mit konventionnellen Instrumenten, doch nach dem Notverkauf des Schlagzeugs entwickelten sie mangels finanzieller Möglichkeiten ein Instrumentarium aus zusammengesuchtem Schrott und Alltagsgegenständen, darunter Stahlteile, Fässer, Hämmer, Bohrmaschinen und Sägen, ergänzt durch eine nicht gestimmte E-Gitarre und Blixa Bargelds markerschütterndes Schreien. Seine oft kryptischen Texte kreisten dabei vor allem um Untergangsfantasien. (Weh, in Emmerling/Weh 2014: 38)

Kollaps war ihr erstes Album und erschien 1981 beim Hamburger Musiklabel ZickZack Records (ZZ65) von Alfred Hilsberg. Der Schlagwerker FM Einheit kam von der Hamburger Punkband Abwärts, als Bassist später Alexander Hacke. In Kollaps erweckt der zweite Song Steh auf Berlin mit repetitivem Schlagwerkset und rauher Stimme eine morbide Faszination an der Stadt und Bilder der Apokalypse. Ein Auszug:

9 Die Transkription des Interviews mit dem dänischen TV-Journalisten Adrian Lloyd Hughes vom 6. September 2014, das an der Universität Kopenhagen aufgezeichnet wurde findet sich im Programmheft zur Live-Performance am 8. November 2014.

10 Im Brecht-Haus in Berlin organisierten Uwe Schütte und Falk Strehlow am 16. Ma 2019 einen Studientag zu den Einstürzenden Neubauten: Andy Spencer (Ohio/USA) und Andrew Hurley (Syndey) referierten über Lament und seine Bezüge zum Dadaismus und Erinnerungsdiskurs.
Ich steh auf Zerfall

Ich steh auf Krankheit

Ich steh auf Niedergang

Ich steh auf Ende

Bacchantischer Gesang und Untergangsbilder passten sehr zu Westberlin am Beginn der 1980er Jahre. Ein Markstein dafür ist gewiss das Festival der Genialen Dilletanten am 4. September 1981, Blixa Bargeld schrieb zum Geleit für dieses Ereignis:

Unsre Musik sind keine Töne mehr, es ist auch nicht wichtig was es für Klänge sind, es ist nur noch wichtig was es ist und noch dazu parteiisch. Die Maschiene [sic] funktioniert, alle sind wir Geiseln. In einem schalltoten Raum gibt es zwei Töne, einen hohen (das Geräusch des arbeitenden Nervensystems) und einen tiefen (den des pulsierenden Blutes) oder umgekehrt. Wir machen keine Fehler mehr, wir werden nichts bei geschlossenem Fenster wiederholen, schrei dich zu Tode. Das ist mehr als richtig. Venceremos. (Bargeld 1982).

Dietrich Diederichsen, Fachmann für Pop-Musik, betont den Gegensatz zwischen der „ehrpusseligen Betulichkeit der altgewordenen 68er Generation“, ihrer Vorliebe für musikalische Virtuosität, und dem neuen Sound West-Berlins:

Im Herbst 1981 stieg im West-Berliner Tempodrom die Große Untergangsshow, Untertitel: Das Festival der Genialen Dilletanten. Es traten Bands und Projekte auf, denen gemeinsam war, dass sie mit verschiedenen Formaten von Noise, Lärm und Über-die-Stränge-Schlagen und Autismus zum Ausdruck bringen wollten, dass sie dem Abendland im Gegensatz zu der so intensiv in Utopien verliebten Vorgängergeneration nicht mehr allzu viel Zeit gaben [...]. (Diederichsen, in Emmerling/Weh 2014: 12-13)

Nicht nur im Tempodrom, sondern auch in Berliner Clubs wie dem SO36 in der Kreuzberger Oranienstraße hörte man die Einstürzenden Neubauten, sie traten auf mit den Frauenbands Malaria! und Neonbabies. Triebfeder für die vierzigjährige Karriere ist sicher die libertäre Macht, sich selbst neu zu finden und erfinden (Hacke 2015). Freiheit bedeutet auch, dass alle Mitglieder der Band individuelle Projekte verfolgen können. Blixa Bargeld war lange Gitarrist bei Nick Cave \& The Bad Seeds (1983-2003), Alexander Hacke komponierte Filmmusik, unter anderem für Fatih Akin in Gegen die Wand (2004). Zu Recht genießen die Einstürzenden Neubauten einen Ruf als défricheurs, oder schlicht auf Deutsch, als Wegbereiter. Standard sind extraordinäre Auftrittsorte: Ödland, Fabriken, Autobahnbrücken und andere industrielle Brachen, aber auch ehemalige Prestigegebäude der Nationalsozialisten oder der abzureißende Staatspalast der DDR sind aufzulisten. Auch bei Theater- und Radioproduktionen mit Peter Zadek, Heiner Müller oder Werner Schwab haben sie mitgewirkt. Commissioned Music heißt Blixa Bargelds erstes Soloalbum (1995) und vereint musikalische Auftragswerke für 
Film und Theater. Schaffen die Einstürzenden Neubauten vor allem avantgardistische Produktionen? Die Bezeichnung als Avantgarde ist heikel, wie Blixa Bargeld im Kopenhagener Interview unterstreicht. Im Programmheft zur Live-Performance liest man in Transkription:

First of all, I have a problem with the term „avant-garde“. [...] it's a military term. It means the guard that runs before the rest of the soldiers and if I want to see myself represented in military terms, I don't want to be a part of that. I want to be one of the deserters. I want to be in the woods maybe. Maybe I want to be a partisan but I don't want to be part of the „Avant-garde“.

Dank der performativen Dimension ihrer Arbeit fassten die Einstürzenden Neubauten Fuß in der zeitgenössischen Kunst, wurden eingeladen zur Biennale de Paris und documenta 7. Mit Auszug/Exodus kreierten sie 1984 eine site-specific performance in der Mojoves-Wüste in Kalifornien, drehten 1985 während einer Tournee in Japan den Film Halber Mensch mit Sogho lishi, in dem Butoh-Tänzer auftraten. Ein sehr medienwirksames Konzert fand am 22. Februar 1986 in Nürnberg statt, und zwar im Goldenen Saal der Zeppelintribüne auf dem Reichsparteitagsgelände. Im März 1989 spielten die Einstürzenden Neubauten auf dem Dach der Fiat-Werke in Lingotto. Eine weitere Performance im öffentlichen Raum war Das Auge des Taifun (Wien, Ringstrasse, 16. Mai 1992), entwickelt von Erich Wonder, den Einstürzende Neubauten und Heiner Müller zum 300. Jahrestag der Gründung der Akademie der Künste in Wien. Zu Beginn der 2000er Jahre schuf die Berliner Band auch ein alternatives Modell zur Finanzierung ihrer Produktionen: Fans wurden aktiv am Produktionsprozess beteiligt. Über dieses Supporter-Projekt holten die Sophiensæle und das Berliner HAU zur Band einen Chor der 100 Stimmen für eine Performance am 4. November 2004 vor dem Abbruch des bereits entkernten Palasts der Republik.

\section{QUELLENARBEIT UND ENTERTAINMENT}

Mit Blick auf die Performance in Diksmuide kann man sich allgemeine Fragen über Auftragswerke stellen. Welche Vorgaben und welches Budget stellt ein Auftraggeber zum gewünschten Werk? Wie viel Freiheit haben Künstler-innen bei der Realisierung? Für Lament hatte die Berliner Musikgruppe freie Hand, doch lagen folgende Eckpunkte zu Grunde: Das Konzert sollte eine Stunde dauern, einen inhaltlichen Bezug zum Ort aufweisen, Passagen sollten in Flämisch aufgeführt werden. ${ }^{11}$

Neben inhaltlichen Aufgaben stellen sich für Künstler·innen auch ökonomische Fragen, insbesondere hinsichtlich des Umfangs des Werkes. Für ein einzelnes Konzert, also wenn es nur ein Konzert in Diksmuide gegeben hätte, wäre eine mögliche Lösung gewesen, einen Mix aus dem Bandrepertoire und einiger pazifistischer Lieder zusammenzustellen,

11 Notiz zum 13. August 2013 auf der Webseite http://fromthearchives.com/en/chronology3.html, zuletzt aufgerufen am 28. Februar 2021. die Absichtserklärung des GoneWest-Programms sah einen Fokus darauf vor. Wie begründete sich nun die Notwendigkeit, ein Album aufzunehmen und zu verkaufen, mit dem die Einstürzenden Neubauten auch lange tourten?

Die Widersprüche sind augenfällig. Einerseits lag der Erste Weltkrieg Blixa Bargeld als Thema nicht „besonders am Herzen", sondern wurde als traumatisch empfunden. Für die Band sei es nicht darum gegangen, familiäre Erinnerungen in das Projekt zu investieren, so der Musiker in einem Interview mit dem Stadtmagazin klenkes.de in Aachen im November 2014. Andererseits war die für das Konzert in Diksmuide versprochene Summe zu niedrig, um die Produktion eines Albums zu rechtfertigen. Es gab gewisse pragmatische Gründe für die Aufnahme des Albums, wie Blixa Bargeld in einem ausführlichen und erhellenden Artikel von Matt Frost in Sound on Sound, einer Fachzeitschrift für Toningenieur $\cdot$ innen, erklärt:

[Recording the album] was a purely economical decision. [...] For the work that we had to put into this performance, it was very clear that we could not finance it with the price that comes with the commission. So we had to revive an existing record contract that was originally made with Mute Records and then ended up as EMI and then ended up as BMG because we didn't want to be bothered with all the work that comes with actually doing the record ourselves. So we revived that record contract and took the advance. It was purely an economical decision. (Bargeld zit. in Frost 2015)

Bei der Genese ist der Faktor Zeit wichtig, die Chronologie der Produktion zeugt von intensiver Arbeit. Nachdem im August 2013 der Auftrag für das Konzert in Diksmuide erteilt wurde, arbeitete Blixa Bargeld mit einem Historiker und einer Literaturwissenschaftlerin an den historischen und literarischen Quellen. Die ersten Aufnahmen begannen laut Booklet im Dezember 2013 und dauerten bis zum September 2014 im Tonstudio andereBaustelle in Berlin-Gesundbrunnen. Im Herbst 2014 wurden die Promo-Versionen an Fachjournalist-innen wie Maxime Lachaud versandt, er interviewte Blixa Bargeld für die Zeitschrift Obsküre am 7. Oktober 2014 (Lachaud 2014), drei Tage später spielte der Radiosender France Culture schon Auszüge aus dem Album, das ab dem 7. November 2014, einen Tag vor der Weltpremiere im Busdepot Diksmuide, in Verkauf ging. Danach tourte die Performance in Deutschland (Aachen, Hannover, Berlin, München), war in Prag, vor frankophonem Publikum (Lausanne, Paris) und in Italien (Bologna, Turin, Rom) zu sehen. In Rom verletzte sich Blixa Bargeld, Konzerte in der Türkei und in Russland wurden abgesagt bzw. verschoben. Nach Weihnachten 2015 gaben die Einstürzenden Neubauten mehrere Konzerte im Berliner Radialsystem (Lament und Greatest Hits). Nach zwei Konzerten beim Festival Dark Mofo in Australien kam Lament noch einmal nach Westflandern zu einem Gratiskonzert unter freiem Himmel und dann in den Berliner Pierre-Boulez-Saal im November 2018.

Eingehende Archivrecherchen kennzeichnen die Kreation. Blixa Bargeld wies in vielen Interviews darauf hin, dass das Album stets mit der in Auftrag gegebenen Aufführung stehe 
und somit immer in Bildern und visuellen Dimensionen erdacht sei. Der Sänger und Texter will überraschen, ihm sei sehr bewusst, dass im Jahr 2014, lange vor dem Konzert in Diksmuide, weitere künstlerische Produktionen entstanden. Man müsse Neues bieten und anspruchsvoll sein, so Blixa Bargeld in der Zeitschrift Sound on sound:

I knew that in England - as well as in Germany and probably several other European countries - the remembrance machinery for 2014 was in full swing. I knew that by the time that we would go on stage in November 2014, we would have almost a year behind us of this remembrance machinery. I had to find some aspect, some corners of knowledge that were not so trampled down. (Bargeld zit. in Frost 2015)

Im Saalheft des Pierre-Boulez-Saals resümierte Blixa Bargeld den Auftrag an seine beiden wissenschaftlichen Zuarbeiter-innen. Klar ausgebreitet habe er,

wonach gesucht werden soll, nach Aspekten, die noch nicht so bekannt und ausgetreten sind - ich wollte nicht einfach nur die Wiederholung von bekannten Dingen wie Grabenkrieg, Matsch und Modder. Schnell wurde mir bei der Arbeit mit den vielen Quellen und den beiden Wissenschaftlern klar, dass es gar nicht mehr nur um den Ersten Weltkrieg, sondern um Krieg ganz allgemein gehen muss.

Was ist das Ziel von Lament? Wehklage oder etwa Unterhaltung? Am 6. November 2014 erschien im Berliner Tagesspiegel ein Interview mit Blixa Bargeld, der die Ursprünge des Projekts und ihre eigenen Erwartungen erklärte. Man bemerkt eine gewisse Distanzierung des Musikers zum Album Lament:

Ich würde es nicht als Neubauten-Album bezeichnen. Aber vor allem sollte es nicht didaktisch sein, was manchmal schwer war - historische Inhalte zu vermitteln, ohne oberlehrerhaft zu wirken. [...] ich bin ja in der Grauzone zwischen Avantgarde und Entertainment tätig. Ich möchte nichts Langweiliges in die Welt setzen, keine reine Belehrung, keine Horrorshow, sondern es soll unterhalten. Frei nach Tom Waits: You have to tell a horrible story beautiful. Das war unser Weg.

Auch wenn der Gedanke an ein unterhaltsames Denkmal überraschen mag, so ist die Erinnungsarbeit konkret und sorgfältig. Stets unterstrich der Musiker, dass man nicht gewahr sei, wie der Erste Weltkrieg wirklich klang. Was sind die wahren Tonarchive? Wo kann man sie finden? Das Booklet des Albums verweist auf Rundfunkarchive, das Militärhistorische Archiv der Bundeswehr in Dresden, das Lautarchiv der Humboldt-Universität zu Berlin und das Ethnologische Museum der Staatlichen Museen zu Berlin. Aber auch Jazz-Aufnahmen fließen in Lament ein, wie Blixa Bargeld im Berliner Kulturmagazin tip vom 6. November erklärte:

Ich habe nach Sachen gesucht, die ich irgendwie musikalisch verwerten konnte. Da gab es einmal die Harlem Hellfighters. Das war ein aus afroamerikanischen Soldaten bestehendes Infanterie-Regiment, dessen Geschichte und

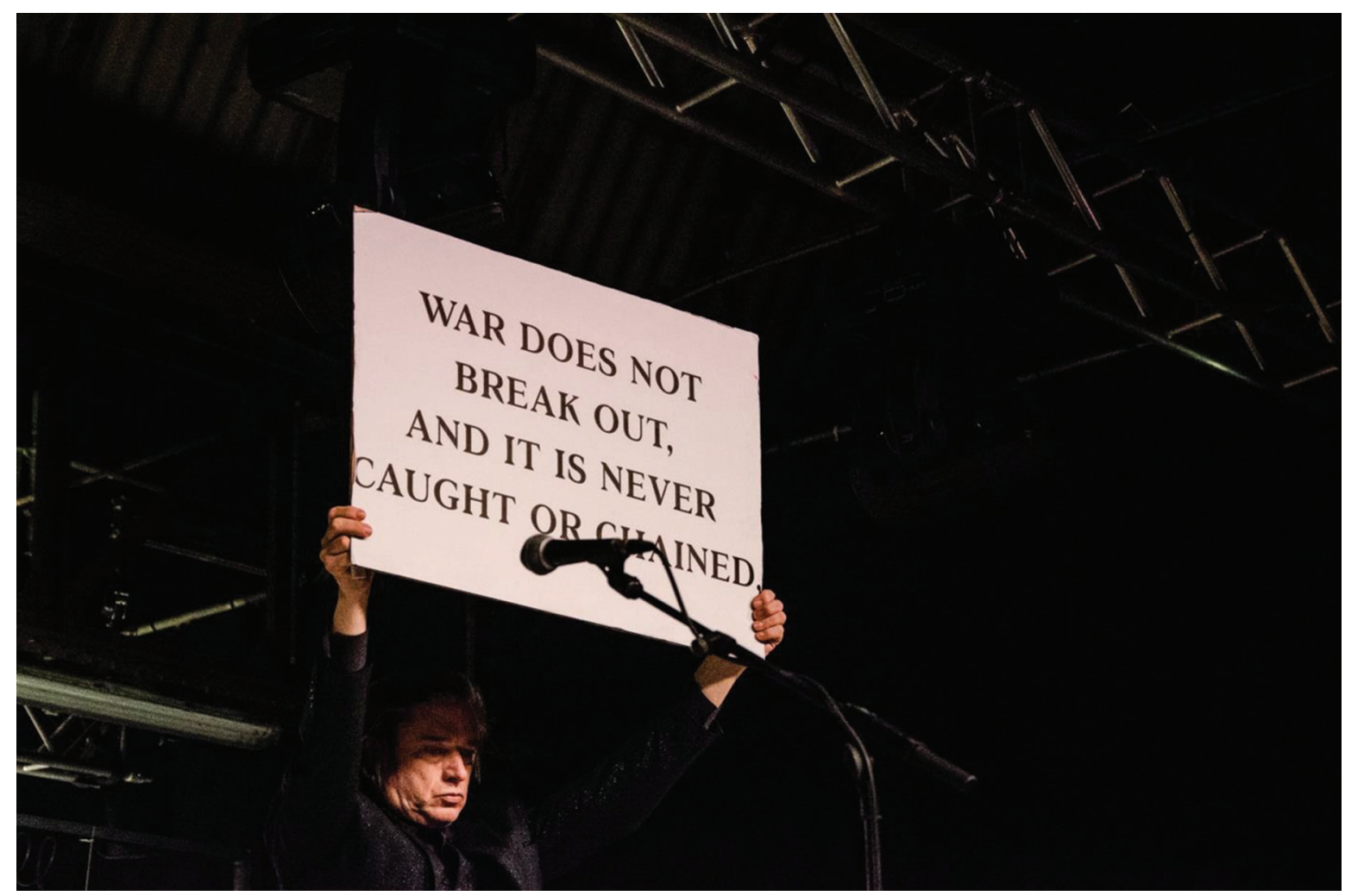

Abb.2: Blixa Bargeld zu Beginn der Performance. Weltpremiere Lament, Busdepot Diksmuide, 8. November 2014. @ Wannes Dewilde. 


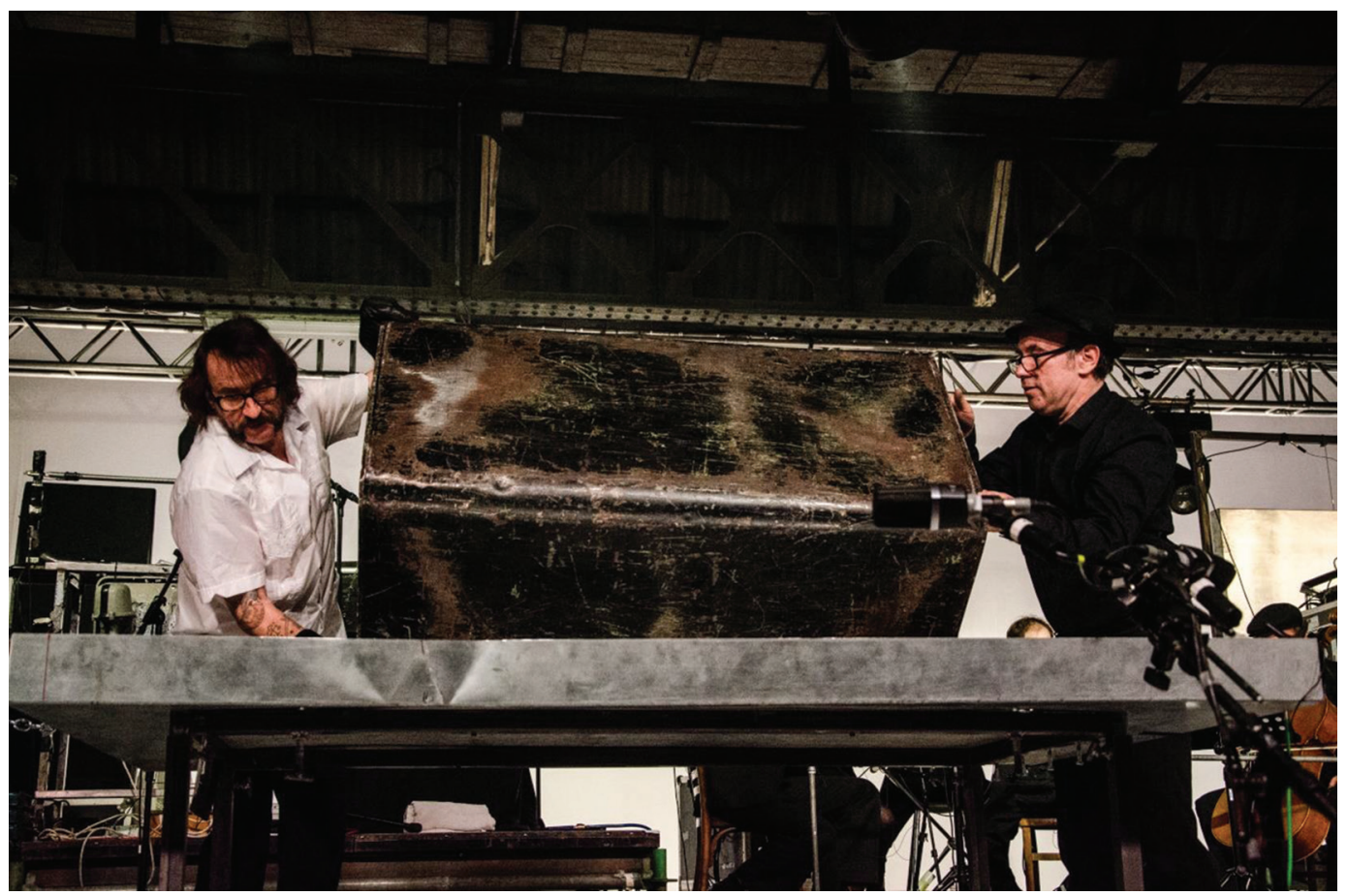

Abb.3: Alexander Hacke (links) und Andrew Unruh am metallischen Schlagwerk, Streichquartett um Jan Tilmann Schade im Hintergrund. Weltpremiere Lament, Busdepot Diksmuide, 8. November 2014. () Wannes Dewilde.

auch Musik in Europa kaum bekannt sind. [... Die Musik der Hellfighters besteht auf Tonträgern], aber erst aus dem Jahr 1919, aufgenommen nach Kriegsende. Die Schwierigkeit war, dass es fast keine Tondokumente aus dem Krieg gibt, weil die Aufzeichnungsverfahren erst am Anfang standen. Es gab die Edison-Walzenrekorder in Berlin, und damals hat das Musikethnologische Institut Aufnahmen von Kriegsgefangenen gemacht und auch die Linguisten an der Humboldt-Universität machten Aufnahmen. Zufälligerweise lag also das einzig existierende Tonmaterial aus dem Ersten Weltkrieg hier in Berliner Archiven.

\section{KOMPOSITION MIT STREICHQUARTETT UND STACHELDRAHTHACKBRETT}

Das Album Lament öffnet mit zwei eng verbundenen Tracks, Kriegsmaschinerie und Hymnen. Der erste dauert fünfeinhalb Minuten, bei den Live-Performances zeigte der Sänger stumm Texttafeln in deutscher oder englischer Sprache zu genau vermerkten Zeiten. Die Texte, hier die erste und die zwei letzten Zeilen in Deutsch, findet man in deutscher und englischer Sprache im Programm bzw. Booklet:

0:00 — Der Krieg bricht nicht aus, war nie gefangen oder angekettet $[\ldots]$

5:07 - Der Krieg bricht nicht aus. Er wartet. Auf ein einziges, tausendfaches:

15:17 [sic] — Hurrah!
Die Dramaturgie ist klar und direkt, nach den nachdenklichen Texttafeln und Hurrah-Rufen geht es direkt über zu patriotischen Hymnen. Blixa Bargeld unterstrich im Interview mit Maxime Lachaud die Komplexität der Parodie im folgenden Teil des Albums, der Hymnen als Titel trägt. Musikalisch seien die Hymnen vieler Kriegsparteien identisch. Die englische Nationalhymne, damals God save the King, stimme klanglich mit der preußischen Hymne Heil dir in Siegerkranz überein, aber auch mit der dänischen, bulgarischen, kanadischen Hymne. Es handele sich um dasselbe Musikstück mit unterschiedlichen Texten, das in einer Reihe von Ländern zu finden ist, die in den Ersten Weltkrieg eingetreten waren (Lachaud 2014). Blixa Bargeld fusionierte verschiedene Versionen, darunter auch Parodien der Hymnen, entlieh Reime aus Heinrich Hoffmanns Bilderbuch König Nußknacker und der arme Reinhold (1851):

\section{Beissen wie Du, wer kann's? \\ Nüsse des Vaterlands \\ Lässt du gewiss nicht ganz. \\ Heil Kaiser Dir!}

Zeilen aus populären Parodien der preußischen Hymne, die schon im Vormärz als Gassenhauer zirkulierten, ${ }^{12}$ schließen den Track Hymnen bei den Einstürzenden Neubauten, wobei die Streicher ins gezupfte Spiel übergehen und der Sänger bissig die Worte artikuliert:

12 Siehe Richter, Lukas (2004 [1969]), Der Berliner Gassenhauer: Darstellung, Dokumente, Sammlung, Münster, Waxmann, 276 und 331. 


\author{
Heil dir im Siegerkranz! \\ Kartoffeln mit Heringsschwanz. \\ Heil Kaiser dir! \\ Friss in des Thrones Glanz \\ Die fette Weihnachtsgans \\ Uns bleibt der Heringsschwanz \\ In Packpapier
}

Nicht größer könnte der Gegensatz sein zwischen jubelnden Untertanen, die an Gräten nagen, während der Kaiser sich am fetten Weihnachtstisch labt. Mit subtilen Mitteln verstehen es die Arrangeure Autorität in Frage zu stellen. Ein eher elektronisch unterlegter Song schließt sich an Hymnen an: The Willy-Nicky-Telegrams, dritter Track in englischer Sprache, montiert die telegraphische Korrespondenz zwischen dem russischen Zaren und dem deutschen Kaiser. Der größte Teil Europas, so Blixa Bargeld, sei 1914 immer noch monarchistisch gewesen, aber alle diese Monarchen hatten Familien, die inzestuös miteinander verbunden waren: Kaiser Wilhelm II. und Zar Nikolaus II. hatten die gleiche Tante, nämlich Victoria, die die Königin von England war. Wenn man sich den Stammbaum ansehe, seien alle Monarchien in Europa miteinander verbunden. Die Hymnen repräsentieren diese Inzucht mit dieser Mischung aus verschiedenen Sprachen, und am Ende kam es zu dieser persiflierenden und satirischen Version der Hymnen (Lachaud 2014).

Der vierte Track, In de loopgraaf, ist in Flämisch aufgenommen, nur Stimme und ein immenses Hackbrett aus Stacheldraht kommen zum Einsatz. Wie auch im siebten Song Acherland erfand Blixa Bargeld, Autor beider Texte, einen fiktiven flämischen Poeten, Paul van den Broeck, der diese Gedichte verfasst haben soll. Im Programm wie im Beiheft zur CD kann man eine spannende fiktionale Biographie entdecken, sie vermischt Imagination und Kritik am Literaturmarkt. Paul van den Broeck nenne sich dieser Dichter, er wäre am 22. Juli 1882 in Antwerpen geboren und wohl im Sommer 1940 in den Pyrenäen bei Pau Verstorbenen. Die geheimnisvolle Biographie schließt mit einem ironischen Blick auf die Kriegsdichterei, so der Text im Booklet:

Except for a small number of surviving poems, little is known about Paul van den Broeck. Although a pacifist (and older than most soldiers), he voluntarily signed up for military service in 1915-16, in spite of his military exemption. [...] One reason why almost none of Van den Broeck's work has survived may in fact be because he himself destroyed much of it. The likelihood of being associated with the „war poetry boom“ (Robert Graves) was almost certainly abhorrent to him.

Peter Vanthygem, Redakteur der belgischen Tageszeitung $D e$ Standaard, ging, im Gegensatz zu vielen Kolleg·innen, dieser Fiktion auf den Grund - mit großer Sorgfalt und Anrufen bei Standesämtern (Vanthygem 2014). Das Spiel mit Namen und Fiktionalisierungen hat Tradition bei den Einstürzenden Neubauten, sich Pseudonyme zu geben ist ein befreiender Akt. Gudrun Gut wie auch Alexander Hacke erinnern sich an Blixa Bargelds Freude, Figuren zu erfinden (Dax/Defcon 2010:
19-23). Schon der Verweis auf den musikalischen Fachbegriff des Viervierteltakts hätte in In de loopgraaf so manche Rezensent·innen hellhörig lassen können:

\section{In de loopgraaf}

Hoe kann ik dansen in $4 / 4$ ?

Hoe kann ik dansen in de smalle grachten?

Ein Stück für Stimmen und Schlagwerk, Der 1. Weltkrieg (Percussion version), eröffnet die B-Seite der Vinylausgabe und ist als "statistische Tanzmusik" konzipiert. Die Musiker konnten schon auf ein gebautes Instrumentarium für viele Titel bei Lament zurückgreifen, bauten aber für diesen Track ein hypnotisches Stück, das auch an afrikanische tribale Musik erinnert, eine große Konstruktion aus Plastikrohren. Sie wurde von Andrew Unruh, Alexander Hacke und Rudolf Moser bespielt. Dazu nannte Blixa Bargeld die Namen der am Krieg beteiligten Länder. Sechs Frauenstimmen gaben Städte und Orte von Kämpfen wie Diksmuide oder Hartmannswillerkopf an. Jedes Land werde durch ein Plastikrohr repräsentiert, so die Anmerkungen im Booklet:

\begin{abstract}
Each country is represented by one pipe, the pipes representing the colonial powers (UK, Germany, etc.) including their colonies and dependencies are represented by a whole set of pipes, starting along the time-line of entering the war until ending their military engagement. Each day is one beat within a bar.
\end{abstract}

Die Musiker wählten als Tempo 120 Schläge pro Minute, dies führte zu einem rund dreizehnminütigen Track. Er zeigt wohl am deutlichsten, wie eine ästhetische Auseinandersetzung mit geschichtlichen Daten, mit Kriegsschauplätzen und mit der Länge der Kämpfe, aussehen kann.

Im Kern des Albums (C-Seite der Vinylversion) steht Lament, eine Sequenz aus drei Sätzen (1. Lament, 2. Abwärtsspirale und 3. Pater peccavi). Hier griffen die Musiker auf alte Musikformen zurück. Das Lamento, das nur als Titel blieb, stelle inhaltliche wie auch strukturelle Probleme, so sei ein Klagelied dazu da, den Feind zu beschuldigen, was nicht der Wunsch Blixa Bargelds gewesen sei (Lachaud 2014). Tonmaterial aus den Berliner Archiven wurde verbunden mit einer achtstimmigen Motette des Renaissancekomponisten Jacobus Clemens Non Papa, der in Diksmuide gestorben war. Aus dem Vokalstück wurde ein wirksam entschleunigtes Arrangement für Streichquartett. Die beiden Tonquellen zu finden, war ein unwiderstehlicher Glücksfall, so Blixa Bargeld im Programmheft zur Live-Performance:

I thought it was such an irresistible coincidence that the German linguistics interviewing the prisoners of war, making them recite Pater peccavi - the prodigal son and there is a renaissance composer buried in Diksmuide who wrote a motet called Pater peccavi. I couldn't resist it.

Das Streichquartett um den Cellisten Jan Tilmann Schade ist hier sehr tragend, dieses Lamento überraschte wohl die 
an metallische Klänge gewöhnten Fans der Einstürzenden Neubauten. Blixa Bargeld und seine Band zeigen im Kernstück des Albums viel Feingefühl für historische Realitäten. Achtsamkeit sei geboten bei der Arbeit mit den Stimmen von Kriegsgefangenen, die von deutschen Wissenschaftlern während des Krieges aufgezeichnet wurden. Das Booklet nennt deshalb die Menschen hinter den Stimmen und den jeweiligen Aufnahmeort. Die historischen Tondokumente aus den Schallarchiven seien sehr zerbrechlich, so Blixa Bargeld im dänischen TV-Interview, das im Programmheft für Diksmuide transkribiert wurde:

I thought it would have been too simple to just use the recordings, like compose music and put recordings on it. So, we performed with these recordings. We used very small speaker cubes, like $5 \mathrm{~cm} \times 5 \mathrm{~cm}$ cubes which acts surprisingly powerful and we played with them like eggs. We played them like raw eggs. [...] I was very, very careful to not use these recordings as some kind of like validation of authencity [...] These are people long dead. These are people that had to speak this under pressure. They were in prison. So, it's a certain delicacy involved in composing something with these kind of ghost voices.

Das Werk erinnert an Vergessene, durchaus kann man eine antimilitaristische Haltung und eine kritische Hinterfragung von Gedächtnisdiskursen im Album Lament finden. Der Song How did I die? schließt die C-Seite vor einer langen Endlosrille. Er greift auf einen Text von Kurt Tucholsky zurück: die Rote Melodie. Bei Tucholsky kommen die toten Soldaten zurück und warnen die Kriegstreiber: „General! General! Wag es nur nicht noch einmal! Es schrein die Toten! Denk an die Roten!"13 Der Song, der das Hauptprogramm von Lament abschließt, lässt eine Landschaft ohne Menschen entstehen. Man hört unter anderem:

Every sign of humanity has been swept away. The woods and roads have vanished like chalk wiped from a board; of the villages nothing remains but gray smears where stone walls have tumbled together. [...] Columns of muddy smoke spurt up continually as high explosives tear deeper into this ulcered area.

Der Schlussrefrain lautet „We didn't die“ (wir sind nicht gestorben). Es gelte den Tod zu bannen, so Blixa Bargeld, ohne dieses Stück am Ende wäre die Performance unausgeglichen geworden. Der Tod komme singend zurück, erklärt der Berliner Musiker: „Sans ce basculement à la fin, la performance se serait terminée sur une touche très triste et déprimante, mais je voulais qu'il y ait de l'espoir à la fin." (Lachaud 2014)

Hoffnungsvoll sind auch die letzten drei Titel des Albums (D-Seite der Vinylausgabe), sie stellen präzise geplante Zugaben dar. Blixa Bargeld singt begleitet vom Streichquartett Sag mir wo die Blumen sind. Der Folksong von Max Colpet und Pete Seeger ist in den Performances visuell mit einer

13 Rote Melodie erschien unter Pseudonym am 3. August 1922 in der Weltbühne.
Hommage an Marlene Dietrich verbunden. Die Trouvaille der Kabarettnummer von Joseph Plauts aus dem Jahr 1926 Der Beginn des Weltkrieges 1914 (Dargestellt unter Zuhilfenahme eines Tierstimmenimitators) gibt sarkastisch Ausblicke auf den Gang der Geschichte, während All of no man's land is ours von den Harlem Hellfighters beschwingt das Album schließt.

Wie könnte man besser an den Ersten Weltkrieg erinnern und elegantes Entertainment bieten? Während das Album in der Musikpresse oft als Meisterwerk, mitunter aber auch als „eigenwillig“, „spannend“ oder „anstrengend“ beschrieben wird, ist die Rezeption der Live-Performances äußerst gemischt. Einige Konzertveranstalter innen waren zwar offen für Experimente, nahmen aber Lament nicht ins Programm. Denn: Wem kann man dieses Album zumuten? Richtet es sich an ein Publikum von Connaisseuses und Connaisseurs, das ein Konzert mit Krach und Noise erwartet? Oder an ein geschichtsinteressiertes Bildungspublikum? In Berlin und München schienen manche Fans unzufrieden zu sein, wie man in Konzertkritiken lesen kann. Ist es ein zu verstörendes Album? Mit zuviel Streichern? Zu bourgeois? Jens Utthoff notierte zum Konzert, das am 11. November 2014 im Tempodrom in Berlin stattfand:

Der Abend lebt wie das Album von den radikalen Brechungen [...]. Das Publikum im ausverkauften Tempodrom hat zumindest zum Teil wohl ein eher übliches Neubauten-Konzert erwartet, falls es das gibt, [...] so jedenfalls konnte man die Zwischenschreie und den nicht immer enthusiastischen Applaus lesen. Vielleicht wäre die Performance an einem anderen Ort, im Theater, besser aufgehoben gewesen als im Rockkonzert-Kontext. Das zeigt auch Bargelds vorsichtige Nachfrage, als er ein weiteres Gedicht [...] rezitiert: „Sind Sie sicher, dass Sie zu einem Schützengrabenlied rhythmisch klatschen wollen?" (taz.de, 12.11.2014)

Saskia Müller, die das Konzert im Münchner Muffatwerk für die Frankfurter Allgemeine Zeitung besprach, sah ebenfalls die beunruhigten und enttäuschten Reaktionen einiger Zuschauer-innen:

Vielen Konzertbesuchern scheint sich allerdings vor allem eine Frage zu stellen, die ein Neubauten-Fan entgeistert stellt: Geht das jetzt etwa zwei Stunden so?! [...] [O]ffenbar entsprach der Abend nicht den allgemeinen Erwartungen. Umso mutiger und beachtlicher ist es, dass die Einstürzenden Neubauten sich an dieses außergewöhnliche Projekt gewagt haben und dass die Verantwortlichen in Westflandern überhaupt auf die Idee kamen, diesen Auftrag an die Berliner Band zu vergeben. (faz. net, 18. November 2014)

Die liminale Arbeit an Abgründen und Brüchen charakterisiert die Musik und Texte der Einstürzenden Neubauten. Und auch die Suche nach Außergewöhnlichem. Das Album Lament beweist dies deutlich. Bleibt noch eine Frage, eine ethische Frage, zum Abschluss: Darf man auf einem Konzert tanzen, wenn das Thema der Erste Weltkrieg ist? 


\section{Auswahlbibliographie}

Bargeld, Blixa (1982), „Zum Geleit“, in Müller, Wolfgang (Hg.), Geniale Dilletanten, Berlin, Merve Verlag, 7.

Bourlet, Michaël (2012), La Belgique et la Grande Guerre, SaintCloud, éditions Soteca 14-18.

Dax, Max / Defcon, Robert (2010 [2005]), Einstürzende Neubauten. Pas de beauté sans danger (traduit de l'anglais et de l'allemand par Gérard Nguyen), Rosière en Haye, Camion Blanc.

Einstürzende Neubauten (2014), Lament, London, Mute Records.

Emmerling, Leonard / Weh, Mathilde (2015), Geniale Dilletanten: Subkultur der 1980er-Jahre in Deutschland / Brillant Dillettantes: Subculture in Germany in the 1980s, Hatje Cantz, Ostfildern.

Frost, Matt (2015), „Einstürzende Neubauten's LAMENT“, Sound on Sound, Cambridge, https://www.soundonsound.com/people/ einsturzende-neubautens-lament, zuletzt aufgerufen am 26 . Mai 2020

Hacke, Alexander (2015), Krach: verzerrte Erinnerungen, Metrolit, Berlin. Kruth, Xavier (2014), „Einstürzende Neubauten. Diksmuide, Belgium (08/11/14)“, http://www.peek-a-boo-magazine.be/en/reviews/ einsturzende-neubauten-diksmuide-belgium-08-11-14/ zuletzt aufgerufen am 2. Juni 2020 .
Lachaud, Maxime (2015), „La Première Guerre Mondiale vue par Einstürzende Neubauten", in La guerre de 14 re-présentée: l'art comme réponse à la guerre (= Caliban. French Journal of English Studies, 53, DOI : 10.4000/caliban.1000 (https://journals.openedition.org/ caliban/1000), zuletzt aufgerufen am 26. Mai 2020).

Müller, Saskia (2014), „Entretien avec Blixa Bargeld: Aspects obscurs de la Grande Guerre", Goethe-Institut Frankreich, (https://www. goethe.de/ins/fr/fr/kul/sup/kw1/20551302.html, zuletzt aufgerufen am 30. Oktober 2019).

Shryane, Jennifer (2009), Evading do-re-mi: destruction and utopia. A study of Einstürzende Neubauten, University of Liverpool.

Uthoff, Jens (2014), „Songs über Schlachtfelder“, taz, 21. November 2014.

Vanthygem, Peter (2014), „Wie is Paul Van den Broeck?“, De Standaard, 28. Oktober 2014, https://www.standaard.be/ cnt/dmf20141028_01347366, zuletzt aufgerufen am 26. Mai 2020)

Offizielle Webseite der Band: www.neubauten.org

Englischsprachige Fanseite mit reichen Informationen zu Konzerten und Diskographie: www.fromthearchives.com 